\title{
Role of Intracranial Cerebrospinal Fluid and brain volume in clinical outcome of stroke patients
}

\author{
B.V. Murlimanju' ${ }^{1}$ Rakesh Mishra², Luis Rafael Moscote-Salazar ${ }^{3}$, Amit Agrawal $^{4}$ \\ ${ }^{1}$ Department of Anatomy, Kasturba Medical College, Manipal Academy of Higher Education, Manipal, Karnataka, India \\ ${ }^{2}$ Department of Neurosurgery, National Institute of Mental Health and Neurosciences \\ (Institute of National Importance), Bengaluru, India \\ 3Center for Biomedical Research (CIB), Cartagena Neurotrauma Research Group, \\ Faculty of Medicine, University of Cartagena, Columbia \\ ${ }^{4}$ Department of Neurosurgery, All India Institute of Medical Sciences, Saket Nagar, Madhya Pradesh, India
}

Many factors govern prognosis in acute ischemic stroke. Studies have implicated the role of volumetric brain and cerebrospinal fluid (CSF) analysis in the outcome of stroke patients (1-4). There is an ongoing debate with arguments in support and against of the protective role of cerebral atrophy in stroke (1-4). Theoretically, brain volume and CSF volumes appear to play a more important role in patients with larger infarct volumes, where more brain volume reduces the space available for cerebral oedema. Also, the functional outcome is an interplay of brain volume and intracranial CSF volume as suggested in studies concerning hydrocephalus and brain tumours $(5,6)$. Measurement of brain volume and intracranial CSF volume requires automated systems. Image-based diagnosis is prone to subjective bias and automated volumetric analysis of CSF volume and intracranial volume can detect cerebral oedema, and differentiate malignant oedema with midline shift from those without in large hemispheric infarcts (7). Brain atrophy has been attributed as a favourable prognostic indicator by many previous studies $(3,7,8)$. However, there are studies which have suggested pre-existing brain atrophy to be a negative predictive factor $(2,4)$. Global cerebral atrophy is associated with long term futile endovascu- lar recanalization and poor recovery gets amplified by the age and infarct volume (4).

Kauw et al. suggested that the ratio of intracranial CSF to the intracranial volume is associated with malignant MCA infarct and when added to the prediction and imaging models, it has prognostic significance (1). Monch et al. have evaluated the role of pre-existing brain volume and intracranial cerebrospinal fluid volume and reported no significant impact in prognosticating clinical outcome in stroke patients (9). Heterogeneity in results in most of the studies is due to the variable population characteristics, for example in the study by Monch et al. (9) rarely patients included had malignant cerebral infarction. Diprose et al. reported their findings in a study of 360 patients of ischemic stroke treated with endovascular thrombectomy (10). Diprose et al. concluded that CSF volume has a strong predictive role in the functional outcome of these patients and speculated that worse outcome in patients with cerebral atrophy could be due to poor reserve (10). They found that there was $35 \%$ lower odds of functional independence and 59\% higher odds of worse outcomes with a $5 \%$ increase in the CSF volume in a multivariate analysis (10). Dhar et al. have suggested that percentage change in the volume of 
CSF in follow up scans may be a more sensitive indicator for cerebral oedema, and neurological worsening as compared to the midline shift (7). On the other side, Chen et al. reported a profound correlation between the midline shift and neurological worsening (11). To this effect, Chen et al. created a normogram model comprising 24 hours CT ASPECT, cisterns effacement, hypertension and recanalization to predict patients at risk after endovascular treatment for stroke in anterior circulation (11).

Brain atrophy due to pre-existing small vessel disease has been found to be associated with 7-day stroke severity and worse mRS outcome at 3 months in patients of acute ischemic stroke treated with endovascular therapy (12). Therefore, it is possible that studies differ due to the diameter of the vessel involved in stroke or presence of additional factors which include white matter hyperintensities, leukoaraiosis etc. Another important point to consider when understanding the studies on brain and CSF volume and its imapact is that most of these studies lack volumetry in the follow up scans of patients with poor functional outcome. Follow up scans and volumetric analysis will clar- ify the occurrence of reperfusion associated cerebral oedema and will help in differentiating poor outcome from futile reperfusion (13). Dhar et al. reported in their study of 738 patients with stroke the importance of following CT scans within 24 hours (14). They suggested that change in CSF volume by $10 \%$ in 24 hours is an early significant indicator of cerebral oedema and decline in neurological outcome (14).

These studies with variable results raise an important concern about whether brain atrophy impacts patients with stroke differently in large vessel disease and small vessel disease. Since acute ischemic stroke is one of the most devastating neurological diseases, more studies to conclude the role of brain volume and intracranial CSF volume on function outcome stroke patients undergoing endovascular interventions is warranted. This 'tau' protein may be significantly related to brain volume (15). Thus, further investigation is required to explore the interaction between brain volume, the volume of intracranial CSF, CSF/ICV ratio, functional outcomes in patients of acute ischemic stroke treated with endovascular therapy and CSF tau concentration.

Conflict of interest: none declared Financial support: none declared

\section{REFERENCES}

1. Kauw F, Bennink E, de Jong $\mathrm{H}$, et al. Intracranial Cerebrospinal Fluid Volume as a Predictor of Malignant Middle Cerebral Artery Infarction. Stroke 2019;50:STROKEAHA119024882.

2. Lee $\mathrm{SH}, \mathrm{Oh} \mathrm{CW}, \mathrm{Han} \mathrm{JH}$, et al. The effect of brain atrophy on outcome after a large cerebral infarction. J Neurol Neurosurg Psychiatry. 2010;81:1316-1321.

3. Park J, Goh DH, Sung JK, Hwang YH, Kang DH, Kim Y. Timely assessment of infarct volume and brain atrophy in acute hemispheric infarction for early surgical decompression: strict cutoff criteria with high specificity. Acta Neurochirurgica. 2012;154:79-85.

4. Pedraza MI, de Lera M, Bos D, et al. Brain Atrophy and the Risk of Futile Endovascular Reperfusion in Acute Ischemic Stroke. Stroke. 2020;51:1514-1521.

5. Mandell JG, Langelaan JW, Webb AG, Schiff SJ. Volumetric brain analysis in neurosurgery: Part 1. Particle filter segmentation of brain and cerebrospinal fluid growth dynamics from MRI and CT images. J Neurosurg Pediatr. 2015;15:113-124.

6. Mandell JG, Kulkarni AV, Warf BC, Schiff SJ. Volumetric brain analysis in neurosurgery: Part 2. Brain and CSF volumes discriminate neurocognitive outcomes in hydrocephalus. J Neurosurg Pediatr. 2015;15:125-132.

7. Dhar R, Yuan K, Kulik T, et al. CSF Volumetric Analysis for Quantification of Cerebral Edema After Hemispheric Infarction. Neurocrit Care. 2016;24:420-427.

8. Beck C, Kruetzelmann A, Forkert ND, et al. A simple brain atrophy measure improves the prediction of malignant middle cerebral artery infarction by acute DWI lesion volume. J Neurol. 2014;261:10971103.

9. Mönch S, Sepp D, Hedderich D, et al. Impact of brain volume and intracranial cerebrospinal fluid volume on the clinical outcome in endovascularly treated stroke patients. J Stroke Cerebrovasc Dis. 2020:104831.

10. Diprose WK, Diprose JP, Wang MTM, Tarr GP, McFetridge A, Barber PA. Automated Measurement of Cerebral Atrophy and Outcome in Endovascular Thrombectomy. Stroke. 2019;50:3636-3638.

11. Chen $X$, Huang $Q$, Deng $Q$, et al. A prediction model of brain edema after endovascular treatment in patients with acute ischemic stroke. Journal of the Neurological Sciences 2019;407.

12. Arba F, Testa GD, Limbucci N, et al. Small vessel disease and clinical outcomes after endovascular treatment in acute ischemic stroke. Neurol Sci. 2019;40:1227-1235.

13. Dhar R. Automated quantitative assessment of cerebral edema after ischemic stroke using CSF volumetrics. Neurosci Lett. 2020; 724:134879.

14. Dhar R, Chen Y, Hamzehloo A, et al. Reduction in Cerebrospinal Fluid Volume as an Early Quantitative Biomarker of Cerebral Edema After Ischemic Stroke. Stroke. 2020;51:462-467.

15. Ott BR, Cohen RA, Gongvatana A, et al. Brain ventricular volume and cerebrospinal fluid biomarkers of Alzheimer's disease. J Alzheimers Dis. 2010;20:647-657. 